\title{
Application of machine learning techniques and empirical mode decomposition for the classification of analog modulated signals
}

\author{
Domenico Luca Carnì ${ }^{1}$, Eulalia Balestrieri ${ }^{2}$, Ioan Tudosa², Francesco Lamonaca² \\ ${ }^{1}$ Department of Computer Science, Modeling, Electronics and System, University of Calabria, Ponte P. Bucci 41C, Rende (CS), Italy \\ 2 Department of Engineering, University of Sannio, Corso Garibaldi 107, 82100, Benevento, Italy
}

\begin{abstract}
In this article, an automatic Analog Modulation Classifier based on Empirical mode decomposition and Machine learning approaches (AMC-EM) is proposed. The AMC-EM operates without a priori information and can recognise typical analog modulation schemes: amplitude modulation, phase modulation, frequency modulation, and single sideband modulation. The AMC-EM uses Empirical Mode Decomposition (EMD) to evaluate the features of the signal for the successive classification by using Machine Learning (ML). In the design of the AMC-EM, the selection of the specific ML technique is performed by comparing, with numerical tests, the performance of the (i) Support Vector Machine (SVM), (ii) $k$-nearest neighbor classifier, and (iii) adaptive boosting, since they are commonly used in the field of signal classification. The tests have highlighted that the SVM, specifically the quadratic SVM, permits the best possible performance concerning classification accuracy, by considering different noise intensities superimposed on the signal. To assess the advantages of the proposal, a comparison with other classifiers available in the literature has been undertaken through numerical tests. Finally, the AMC-EM is experimentally evaluated, and the experimental results agree with those of the simulation.
\end{abstract}

Section: RESEARCH PAPER

Keywords: classification of analog modulated signals; empirical mode decomposition; machine learning technique)

Citation: Domenico Luca Carnì, Eulalia Balestrieri, Ioan Tudosa, Francesco Lamonaca, Application of machine learning techniques and empirical mode decomposition for the classification of analog modulated signals, Acta IMEKO, vol. 9, no. 2, article 11, June 2020, identifier: IMEKO-ACTA-09 (2020)-02-11

Editor: Dušan Agrež, University of Ljubljana, Slovenia

Received March 12, 2020; In final form May 30, 2020; Published June 2020

Copyright: This is an open-access article distributed under the terms of the Creative Commons Attribution 3.0 License, which permits unrestricted use, distribution, and reproduction in any medium, provided the original author and source are credited.

Corresponding author: Eulalia Balestrieri, e-mail: eubal@unisannio.it

\section{INTRODUCTION}

Communication systems typically use different analog and digital modulation techniques to send information between two or more apparatuses.

The monitoring and classification of analog communication signals play an important role in military and civilian applications regarding the monitoring of non-authorised transmitters; electronic surveillance; and cognitive radio and reconfigurable communication systems [1][2].

In some of these applications, after the detection of the presence of a modulated signal in the spectrum [3][4], the automatic classification of the modulation scheme without a priori information is needed for the demodulation of the signal.

The classification methods proposed in the literature can be divided into two main groups: decision theoretic and statistical pattern recognition.
In the first group, the classification is based on the information obtained by signal enveloping characteristics, statistical moments, and phase trend [5]-[7]. Usually, the decision theoretic classifiers require empirical evaluation of thresholds that are compared with the estimated parameters of the signal or, in any case, some previous knowledge about the detected signal.

It is noteworthy that the empirical thresholds are evaluated in specific operating conditions, and as a consequence, the effectiveness of the classifier cannot be warranted for new cases.

Finally, the classification performance of the decision theoretic methods typically decreases along with the increasing of the amplitude of the noise superimposed on the signal.

In the second group, the classification is obtained by the pattern recognition of some features estimated in the time or frequency domain on the signal under investigation [1][2][8]-[16]. Moreover, the classification performance of the statistical pattern recognition methods typically decreases along with the increasing 
of the amplitude of the noise superimposed on the input signal due to the variability of the features.

The automatic modulation scheme classification methods proposed in the literature therefore present important limitations due to the requirement to have some previous knowledge about the detected signal, the dependence of the classifier effectiveness on specific operating conditions, and the influence of the amplitude of the noise superimposed on the signal on the classifier performance.

In this article, a new Analog Modulation Classifier (AMC) relying on the joint utilisation of Empirical Mode Decomposition (EMD) and Machine Learning (ML) techniques is proposed to overcome the limitations of the existing classification methods. The AMC-EM estimates a set of features based on the analysis of the signal under investigation and uses these features as an input for a traditional classifier based on opportunely trained ML.

The features are extracted by the EMD [17]-[24] of the input signal. The EMD is chosen for the feature estimation because, differently to other decomposition methods based on Fourier or Wavelet, it does not need a basis defined a priori. So, the EMD is suitable for non-linear and non-stationary signals that are typical in telecommunications. Moreover, each component obtained by the EMD is related to a different band of the signal, and as a consequence, it is influenced in a different way to the superimposed noise. This allows for the acquisition of some components that are influenced by the noise. Concerning the traditional classifier, which is based on ML, different techniques are compared by numerical tests in order to establish the one that ensures the best classification accuracy in different operating conditions.

In this article, the operating conditions that are taken into account are (i) the inaccurate carrier frequency estimation of the signal under examination and (ii) different levels of superimposed noise.

The ML techniques taken into account are those that are the most commonly used in the field of signal classification: (i) the Support Vector Machines (SVMs) [1][25] with linear, quadratic, and cubic kernels, (ii) the k-Nearest Neighbor $(\mathrm{kNN})$ classifier [25], and (iii) adaptive boosting [25].

The signal modulation schemes considered in this paper are Amplitude Modulation (AM), Phase Modulation (PM), Frequency Modulation (FM), and Single-Sideband (SSB) modulation.

The paper is organised as follows: in order to make the article self-contained and to declare the symbols used to define the signals, in Section 2, basic knowledge about the analog modulation schemes is briefly reported. The EMD and the ML techniques are summarised in Section 3 and Section 4, respectively. In Section 5, the signal generation parameters and the features that require estimation are highlighted, and a comparison of the training results of different ML techniques is provided.

In Section 6, the AMC-EM is compared with other classifiers proposed in the literature by numerical tests, and its experimental evaluation is presented. In the last Section, conclusions are drawn.

\section{ANALOG MODULATION TECHNIQUES}

The analog modulation techniques codify the information that is to be transmitted in the amplitude, frequency, or phase modifications of the carrier signal, which is based on a sinewave characterised by the frequency $f_{c}$.
The general form of an analog modulated signal is expressed by the function:

$$
s(t)=x(t) \cos \left(2 \pi f_{c} t+m(t)+\Theta\right)
$$

where $x(t)$ and $m(t)$ are respectively the amplitude and the phase modification codifying the information, and $\Theta$ is the initial phase.

The parameters of this form are imposed according to the particular modulation technique.

In the case of AM, the information is codified only in the amplitude of the carrier signal, and no modification is introduced in its phase.

Then, Equation (1) is modified as follows:

$s(t)=\left[1+i_{m} x(t)\right] \cos \left(2 \pi f_{c} t\right)$

with $i_{m}$ being the modulation index and $x(t)$ the information that is to be transmitted.

In the PM case, the information is codified as an instantaneous phase variation of the carrier signal, while the amplitude of the carrier signal remains constant.

Therefore, the modulated signal can be expressed as:

$$
s(t)=\cos \left(2 \pi f_{c} t+k_{p} m(t)+\Theta\right)
$$

where $m(t)$ is the information that is to be transmitted, and $k_{p}$ is the deviation constant.

In the FM case, the information is encoded in the instantaneous frequency of the carrier signal.

In particular, the deviation of the carrier frequency with respect to its centre frequency is proportional to the amplitude of the information that is to be transmitted.

To obtain this result, the information is integrated with respect to time and is added to the carrier frequency. The resulting signal can be expressed as:

$$
s(t)=\cos \left(2 \pi f_{c} t+\Delta f \int_{-\infty}^{t} m(\tau) d \tau\right)
$$

where $\Delta f$ is the frequency deviation. By considering $m(t)$, bounded in the range $\pm 1, \Delta f$ represents the maximum variation of the instantaneous frequency of the modulated signal with respect to the carrier frequency.

The SSB modulation is a particular AM whereby the bandwidth of the signal is reduced. To obtain this result, Equation (1) is modified as follows:

$$
s(t)=x(t) \cos \left(2 \pi f_{c} t\right) \mp \hat{x}(t) \sin \left(2 \pi f_{c} t\right)
$$

where $\hat{x}(t)$ is the Hilbert transform of $x(t)$.

\section{EMPIRICAL MODE DECOMPOSITION} 24].

The EMD is the basis of the Hilbert-Huang Transform [17Through the EMD, a signal is decomposed in a set of functions called Intrinsic Mode Functions (IMFs) and in a monotone residue. The IMFs are characterised by slow amplitude variations with respect to the oscillation period.

Differently to other decomposition approaches, such as Fourier and Wavelet, the EMD does not have a prefixed basis; therefore, the EMD allows for the acquisition of a highly efficient decomposition.

The main drawback of the EMD is the unpredictable number of the obtained IMFs. As a consequence, it is necessary to establish a suitable fixed number of IMFs to be taken into account for classification purposes. On the one hand, the 
number of IMFs must be sufficiently high so as to allow the evaluation of a sufficient number of features for the ML classifier. On the other hand, it must be sufficiently small so as to be suitable for all the kinds of signal modulation schemes considered in this study.

In order to present the theoretical tools that justify the selection of this number and to make the article self-contained, the criteria used by the EMD to evaluate each IMF and the implemented algorithm are described here.

Each IMF satisfies two conditions [26]:

1. The number of local maxima, local minima, and zero crossings are equal or differ at most by one in the observation interval. This condition ensures that the local maxima and minima are positive and negative, respectively.

2. The mean value of the envelope defined by the local maxima and the envelope defined by the local minima is zero - at any time instant. This ensures that the instantaneous frequency does not have unwanted fluctuation due to the asymmetric waveform.

By considering that $s(t)$ is decomposed in a finite number $N$ of IMF, the input signal can be expressed as:

$$
s(t)=\sum_{i=1}^{N} \mathrm{IMF}_{\mathrm{i}}(\mathrm{t})+r(t)
$$

where $r(t)$ is the residue. The steps of the EMD algorithm are presented in Figure 1, and they are explained as follows:

a. Detect the local extrema of the input signal $s(t)$, as the samples that are larger than four of its neighbouring samples.

b. Fit through cubic splines the upper and lower extrema to obtain the upper and lower envelope.

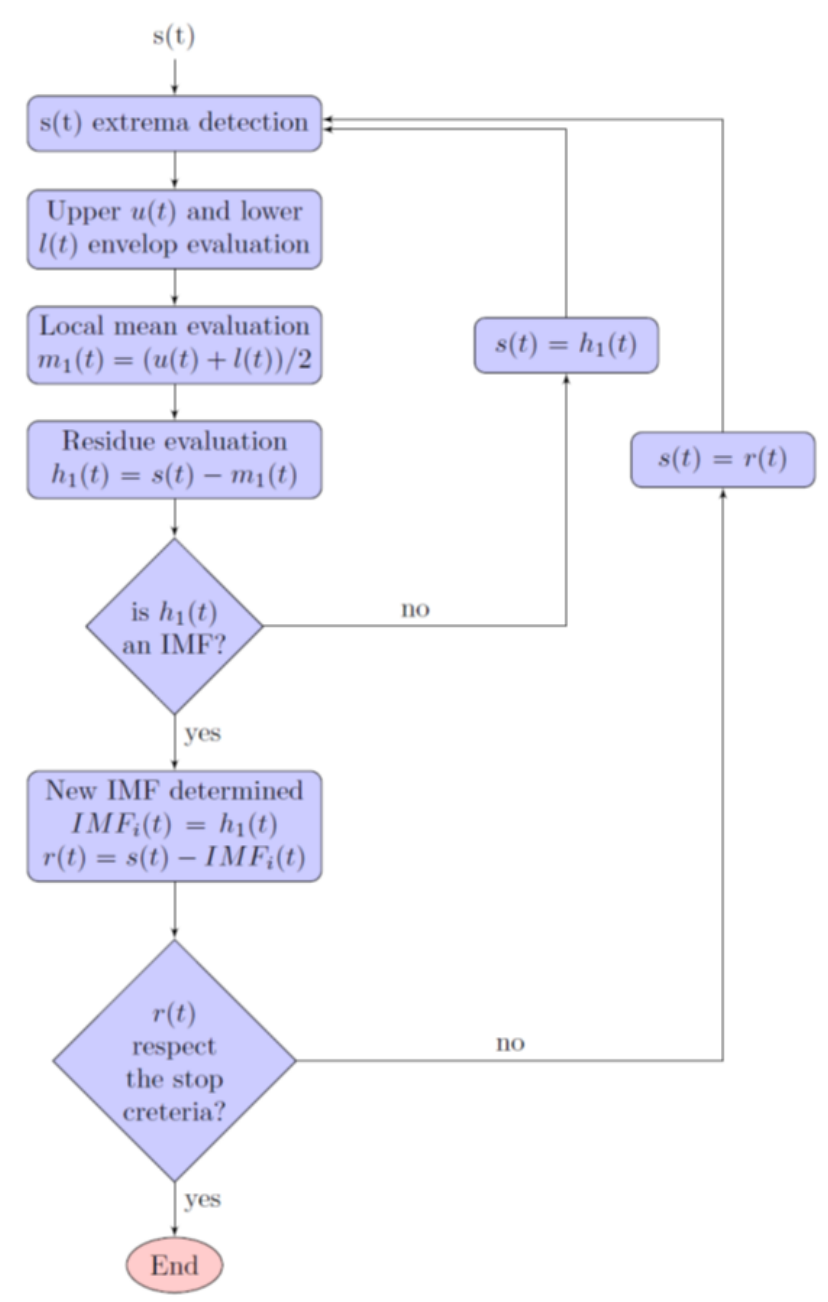

Figure 1. Flowchart of the EMD algorithm.
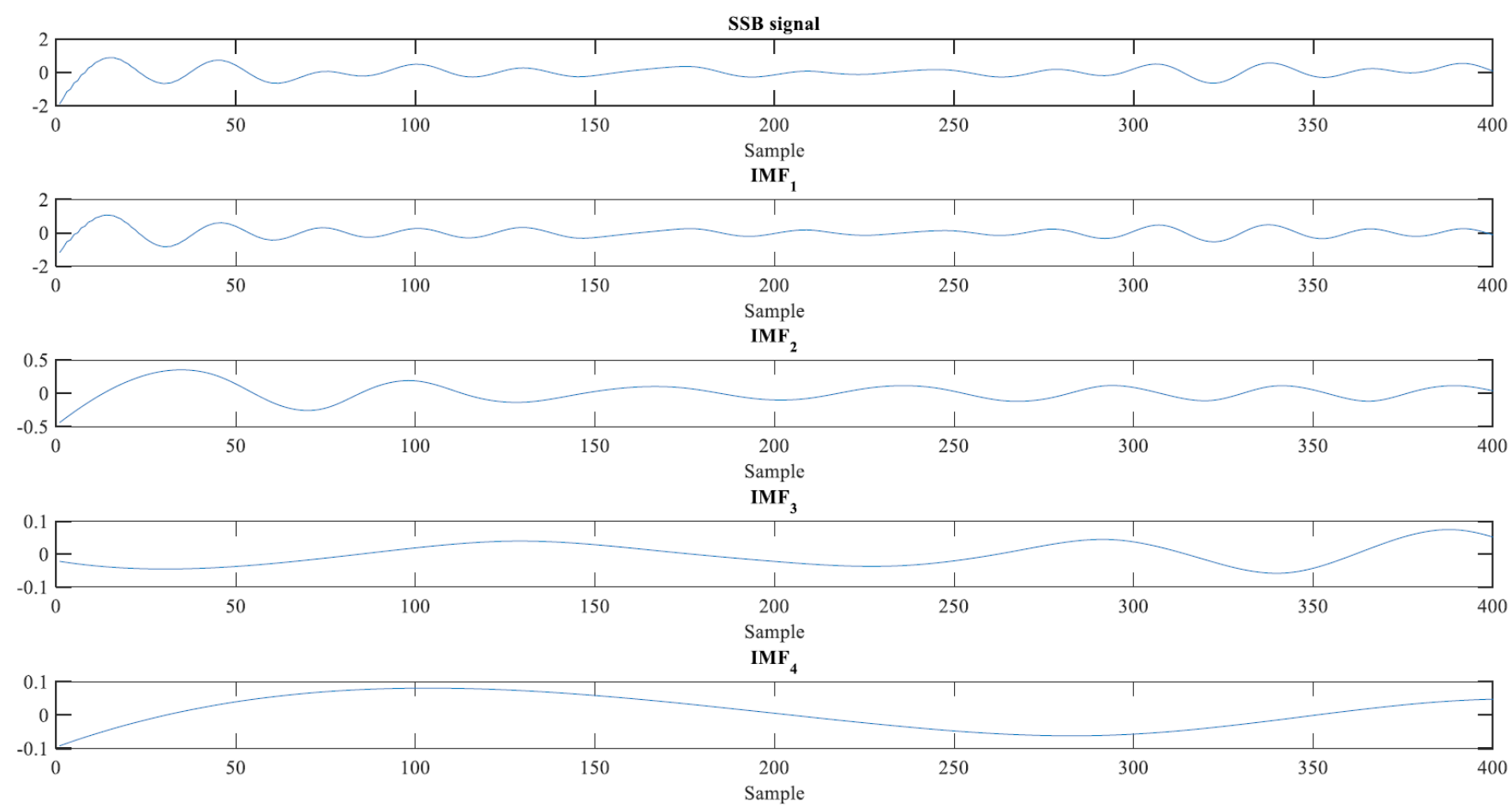

Figure 2. Trend of an SSB modulated signal and its first four IMF components. 
c. Evaluate the mean value $m_{1}(t)$ of the upper and lower envelope point by point.

d. Define $h_{1}(t)=s(t)-m_{1}(t)$.

e. If the two IMF conditions are satisfied by $h_{1}(t)$, then this last one is an IMF. If they are not thus satisfied, then repeat from step (a) by considering $h_{1}(t)$ as the input. Subtract from the input signal $s(t)$ all the evaluated IMF. If the residue has at least two extrema, repeat the procedure from step (a) on the residue for the evaluation of another IMF.

\section{MACHINE LEARNING TECHNIQUES}

The ML includes algorithms designed to learn some of the data features from a dataset automatically [27]-[29].

ML theory proposes different techniques that can be classified as supervised or unsupervised. In supervised ML algorithms, the learning process requires the class and the features of each sample used for training. Once the algorithm learns from the training samples, it can be used on unclassified samples. Conversely, unsupervised learning algorithms tries to learn from unclassified samples (used for training) how to classify them.

This article is focused on the most commonly used supervised learning techniques for signal classification [25]: SVMs, the kNN classifier, and adaptive boosting.

The SVM [1][25] is a supervised learning algorithm that determines the separation of hyperplanes for the classification of different classes of data. The criteria for determining the hyperplanes is typically the maximisation of the margin among the classes and the minimisation of the classification error.

In the case that the input data are not linearly separable, it is possible to map the data in a higher-dimensional feature space by using a nonlinear SVM (for example, quadratic or cubic ones).

The SVM can be advantageously used in the convex optimisation problem i.e. the classification. In fact, the SVM provides a unique solution. Moreover, the classification is performed in the input space by using a kernel function. This also provides an efficient computation in the case of a large set of features.

With $\mathrm{kNN}$, the classification of the input data is based on the closest training example in the feature space [25]. A data is classified on the basis of the majority vote of its $\mathrm{kNNs}$, where the number of neighbours, $\mathrm{k}$, is selected a priori [25].

The neighbours are taken from a dataset with known classification, and the common value of $\mathrm{k}$ is 1 , for which the allocation is made to the nearest neighbour. The main advantage of $\mathrm{kNN}$ is the computation time, which is lower than other ML techniques.

Adaptive boosting is a ML approach that combines many relatively weak and inaccurate rules (classifiers) to create a highly accurate prediction [30]. Iteratively, for each estimated classifier, a score is assigned and linearly combined with the other ones to obtain the final classifier.

\section{SIGNAL GENERATION AND FEATURE EXTRACTION}

The set of signals used to train the ML techniques includes both real and simulated signals.

In particular, in the following section, the case of voice signals is considered as a practical example. The band of the signals is limited to $4 \mathrm{kHz}$, which is the typical single voice-frequency transmission channel bandwidth, by means of a 9th-order lowpass Butterworth filter. The real signals are obtained by modulating audio conversation. Instead, the simulated signals, as suggested in [1], are generated by means of a first-order autoregressive process:

$$
y\left(k T_{s}\right)=0.95 y\left((k-1) T_{s}\right)+n\left(k T_{s}\right)
$$

where $T_{s}$ is the sampling period, and $n\left(k T_{s}\right)$ is a random Gaussian generator. The sampling frequency for the signals is $80 \mathrm{kHz}$.
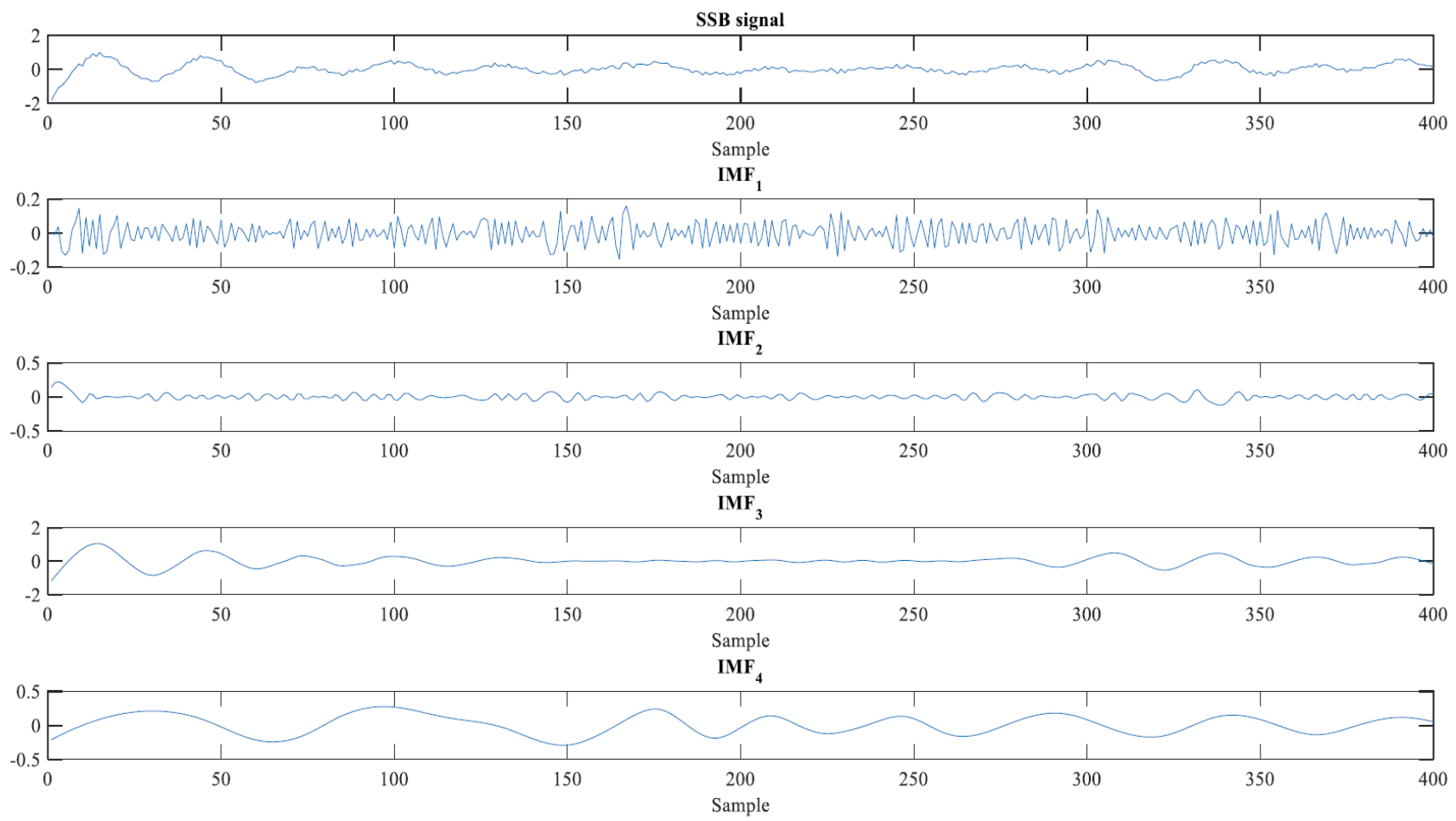

Figure 3. Trend of a noisy SSB modulated signal and its first four IMF components. 
Both the real and the simulated signals are modulated by using the schemes described in Section 2.

In order to obtain the features to train the classifiers and to ensure that these features include the case of inaccurate evaluation of the carrier frequency, for each modulation technique, a set of 650 modulated signals is considered.

The set comprises 50 real signals and 600 simulated signals. By directly considering the intermediate frequency band, the intermediate carrier frequency used to generate the signals varies from $5 \mathrm{kHz}$ to $5.5 \mathrm{kHz}$. This carrier frequency range permits the acquisition of the resulting signals with a low sampling frequency. Finally, the modulated set of signals is affected by arbitrary white Gaussian noise with a Signal-to-Noise Ratio (SNR) included in the range $[-5,65] \mathrm{dB}$.

To establish the number of IMFs for the training of the ML techniques, it is considered that the decomposition process preliminarily isolates the high-frequency mode, and successively, with each step representing a lower-frequency mode. In fact, in Figure 2, the case of an SSB modulated signal and its first four decomposition IMFs is shown.

The decomposition can change in terms of its dependence on the level of noise superimposed on the signal. Figure 3 shows the decomposition of the SSB modulated signal shown in Figure 2 with superimposed noise. The noise makes the $\mathrm{IMF}_{1}$ characterised by higher variability on its trend compared with the one shown in Figure 2 because $\mathrm{IMF}_{1}$ mainly represents the noise. Conversely, the other IMF components show a similar trend to the corresponding ones in Figure 2.

For this reason, for the training of the ML techniques, features extracted from the first three IMF components of the modulated signal affected by different values of noise are considered.

The features extracted from the IMF for the training of the ML techniques are:

- Standard deviation: the second-order moment that measures the variability of the data around the mean value, defined as:

$$
\sigma_{\text {IMF }_{\mathrm{i}}}=\sqrt{\frac{1}{\mathrm{~N}} \sum_{\mathrm{k}=1}^{\mathrm{N}}\left(\mathrm{IMF}_{\mathrm{i}}(\mathrm{k})-\overline{\mathrm{IMF}}_{\mathrm{i}}\right)^{2}}
$$

where $N$ is the number of samples and $\mathrm{IMF}_{1}$ is the mean value of the $i$-th IMF component.

- Skewness: The third-order moment that represents the asymmetry degree of the distribution of the value around the mean value of the IMF, defined as:

$$
\mathrm{S}_{\mathrm{IMF}_{\mathrm{i}}}=\frac{1}{\mathrm{~N}} \sum_{\mathrm{k}=1}^{\mathrm{N}}\left(\frac{\operatorname{IMF}_{\mathrm{i}}(\mathrm{k})-\overline{\mathrm{IMF}}_{\mathrm{i}}}{\sigma_{\mathrm{IMF}_{\mathrm{i}}}}\right)^{3}
$$

- Kurtosis: the fourth-order moment that describes the flatness of the IMF distribution with respect to the normal distribution, defined as:

$$
\mathrm{K}_{\mathrm{IMF}_{\mathrm{i}}}=\frac{1}{\mathrm{~N}} \sum_{\mathrm{k}=1}^{\mathrm{N}}\left(\frac{\mathrm{IMF}_{\mathrm{i}}(\mathrm{k})-\overline{\mathrm{IMF}_{1}}}{\sigma_{\mathrm{IMF}_{\mathrm{i}}}}\right)^{4}
$$

Moreover, in order to better identify the phase modulation, the utilised parameter is the standard deviation of the instantaneous frequency of the IMF component, defined as:

$$
\begin{aligned}
\sigma_{D_{p}} \mathrm{IMF}_{i} & =\left[\frac{1}{\mathrm{~N}}\left(\sum_{\mathrm{k}=1}^{\mathrm{N}} \operatorname{angle}\left(\operatorname{hilbert}\left(\mathrm{IMF}_{i}\right)\right)^{2}\right)\right. \\
& \left.-\ldots\left(\frac{1}{\mathrm{~N}} \sum_{\mathrm{k}=1}^{\mathrm{N}} \operatorname{angle}\left(\operatorname{hilbert}\left(\mathrm{IMF}_{i}\right)\right)\right)^{2}\right]^{\frac{1}{2}}
\end{aligned}
$$
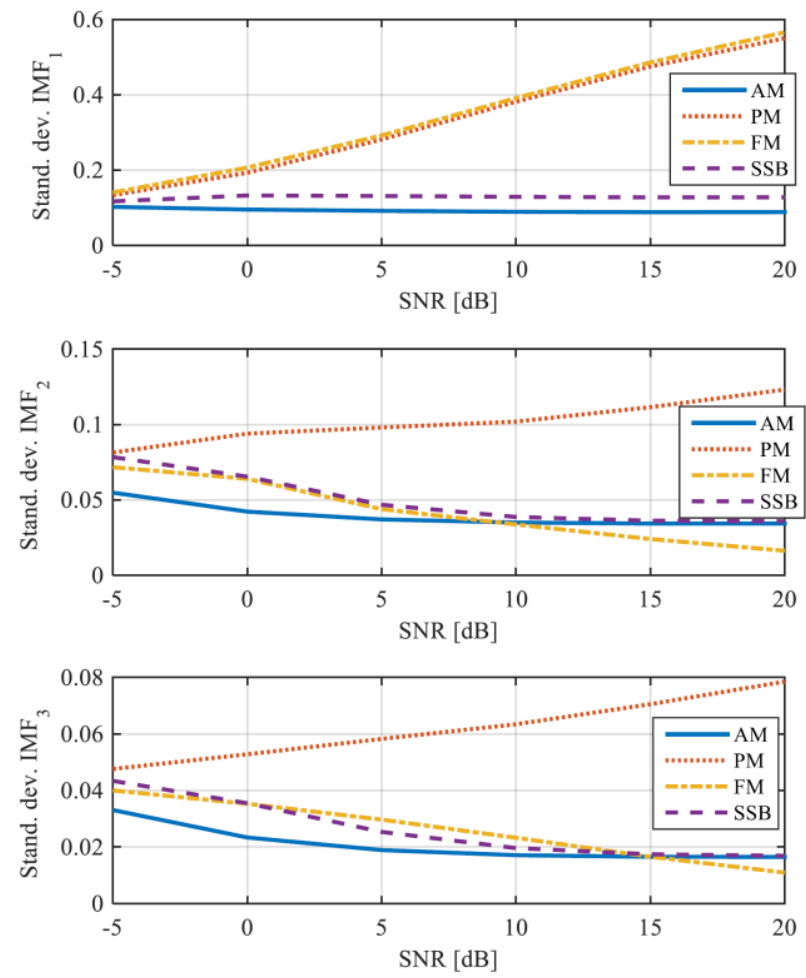

Figure 4. Mean value of the standard deviation for AM, PM, FM, and SSB modulation from the first three IMFs.
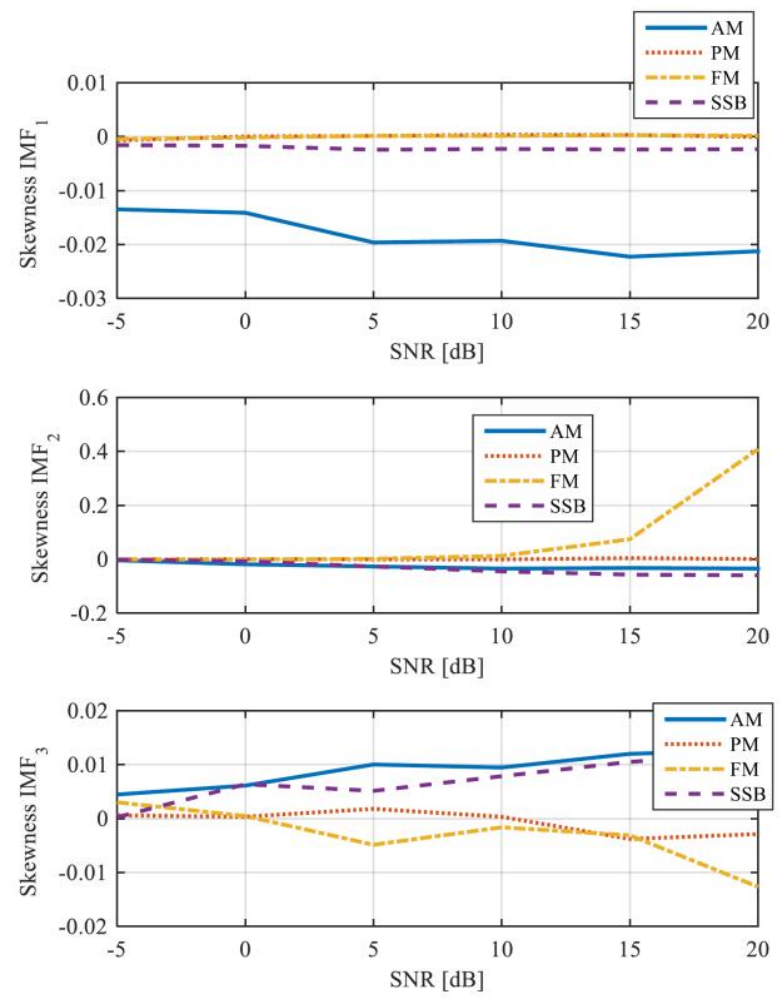

Figure 5. Mean value of the skewness for the modulation AM, PM, FM, and SSB from the first three IMFs. 
In order to reduce the number of parameters used in the classification permitting the correct classification, the mean value of each parameter versus the SNR is considered.

Figure 4 shows the mean value of the standard deviation for the considered modulation schemes versus the SNR in the case the carrier frequency is $5 \mathrm{kHz}$. The figure highlights that the first three IMFs determine different regions in the feature space for the classification of each modulation scheme. The trend of the parameters is due to the spectrum distribution of the input signal, of the noise, and to the band represented by the IMF. The increasing of the noise level reduces the IMF band that will include more noise than signal.

Naturally, these parameters are insufficient for the classification, particularly for a low value of SNR. In fact, the separation between the modulations is small, and the fluctuation of the parameters can generate a misclassification.

Figure 5 shows the mean value of the skewness for the considered modulation schemes versus the SNR. In this case, the trend of the skewness of $\mathrm{IMF}_{1}$ for the different modulation schemes shows a different range of value, particularly for the $\mathrm{AM}$, with minimal influence from the noise. Instead, the other components do not show the same characteristic; therefore, they are not considered as being between the parameters for the training and classification of the ML techniques.

A similar analysis is performed for the mean value of the kurtosis of the IMF of the modulated signals versus the SNR showed in Figure 6. In this case, the kurtosis value of $\mathrm{IMF}_{1}$ shows a separation between the different modulation and is used for the classification. The other decompositions do not show a separation among the modulations, particularly for a low SNR value.

Finally, Figure 7 shows the mean value of $\sigma_{D P}$ for the modulation scheme under consideration versus the SNR value. This figure highlights that all the three IMFs furnish information that can be used for the classification. This parameter is particularly important in the case that a carrier frequency error is present on the signal under examination. Then, the total number of features used in the ML algorithm is 12 .

\section{NUMERICAL AND EXPERIMENTAL TESTS}

The feature extraction and classification systems are implemented in the MATLAB environment. The number of samples used for the classification feature estimation is 20,000 , which represents an observation time of $0.25 \mathrm{~s}$ [1]. The training set of signals is generated as previously described, and the confusion matrix for the considered ML techniques is shown in Figure 8 . The diagonal elements represent the modulated signals correctly classified. Instead, the values outside the diagonal are the misclassified signals.

As evidenced, the linear, quadratic, and cubic SVMs and the $\mathrm{kNN}$ have an overall classification accuracy of $100 \%$. Instead, the adaptive boosting method completely misclassifies the FM signals - recognised as PM. For this reason, in the successive analysis, the adaptive boosting ML technique is not considered.

In order to verify the behaviour of the ML techniques with signals not included in the training set, for each modulation scheme, a test set of 200 signals is generated with a carrier frequency included in the range $[5.0,5.5] \mathrm{kHz}$, and with the SNR equal to $5 \mathrm{~dB}$. Figure 9 shows the confusion matrix for these signals: for the quadratic SVM, the overall classification accuracy continues to be $100 \%$; it decreases to $99.9 \%$ for the linear and cubic SVMs and to $99.5 \%$ for the $\mathrm{kNN}$.
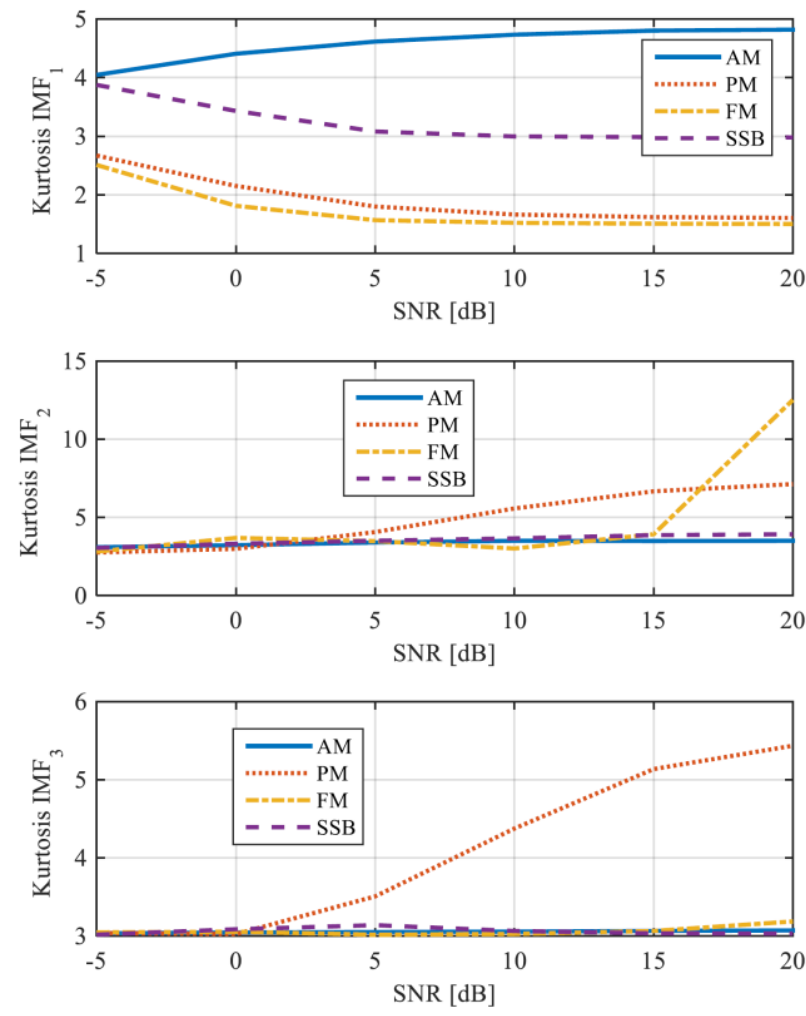

Figure 6. Mean value of the kurtosis for the AM, PM, FM, and SSB modulation from the first three IMFs.
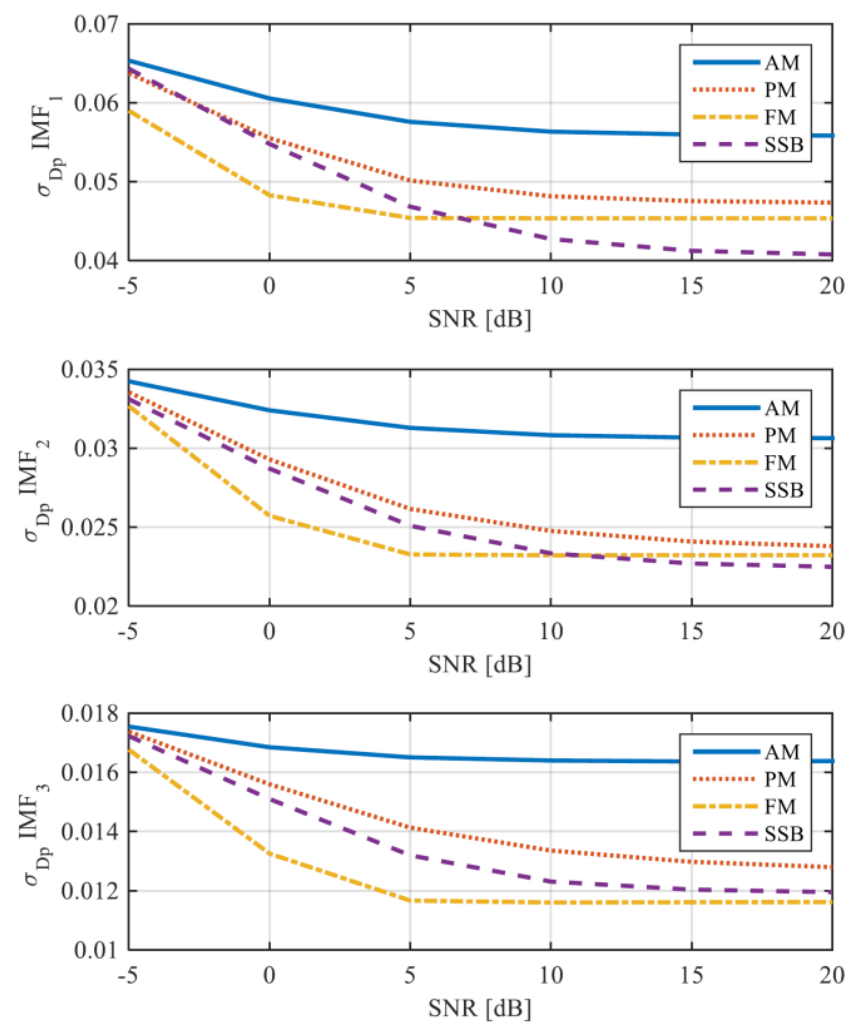

Figure 7. Mean value of $\sigma_{D p}$ for the modulation $A M, P M, F M$, and SSB modulations from the first three IMFs.

\subsection{Comparison with other methods}

In order to assess the advantages of the proposed classifier, AMC-EM is compared with another classifier available in the literature [15] that is capable of classifying the same modulation schemes as the proposed one. 


\begin{tabular}{|c|r|r|r|r|}
\hline \multirow{2}{*}{ True Modulation } & \multicolumn{5}{|c|}{ Predicted Modulation by SVM linear } \\
\cline { 2 - 5 } & AM & \multicolumn{1}{c|}{ PM } & \multicolumn{1}{c|}{ FM } & \multicolumn{1}{c|}{ SSB } \\
\hline AM & 650 & & & \\
\hline PM & & 650 & & \\
\hline FM & & & 650 & \\
\hline SSB & & & & 650 \\
\hline
\end{tabular}

\begin{tabular}{|c|r|r|r|r|}
\hline \multirow{2}{*}{ True Modulation } & \multicolumn{5}{|l|}{ Predicted Modulation by SVM quadratic } \\
\cline { 2 - 5 } & AM & PM & \multicolumn{1}{c|}{ FM } & \multicolumn{1}{c|}{ SSB } \\
\hline AM & 650 & & & \\
\hline PM & & 650 & & \\
\hline FM & & & 650 & \\
\hline SSB & & & & 650 \\
\hline
\end{tabular}

\begin{tabular}{|c|r|r|r|r|}
\hline \multirow{2}{*}{ True Modulation } & \multicolumn{5}{|c|}{ Predicted Modulation by SVM cubic } \\
\cline { 2 - 5 } & AM & \multicolumn{1}{|c|}{ PM } & \multicolumn{1}{c|}{ FM } & \multicolumn{1}{c|}{ SSB } \\
\hline AM & 650 & & & \\
\hline PM & & 650 & & \\
\hline FM & & & 650 & \\
\hline SSB & & & & 650 \\
\hline
\end{tabular}

\begin{tabular}{|c|r|r|r|r|}
\hline \multirow{2}{*}{ True Modulation } & \multicolumn{5}{|c|}{ Predicted Modulation by kNN } \\
\cline { 2 - 5 } & AM & \multicolumn{1}{|c|}{ PM } & \multicolumn{1}{c|}{ FM } & \multicolumn{1}{c|}{ SSB } \\
\hline AM & 650 & & & \\
\hline PM & & 650 & & \\
\hline FM & & & 650 & \\
\hline SSB & & & & 650 \\
\hline
\end{tabular}

\begin{tabular}{|c|r|r|l|l|}
\hline \multirow{2}{*}{ True Modulation } & \multicolumn{4}{|c|}{ Predicted Modulation by adaBoost } \\
\hline & AM & PM & FM & SSB \\
\hline AM & 650 & & & \\
\hline PM & & 650 & & \\
\hline FM & & 650 & & \\
\hline SSB & & & & 650 \\
\hline
\end{tabular}

Figure 8. Confusion matrix of the training set for the $\mathrm{ML}$ techniques taken into consideration.

Other available classifiers are specific to single modulation and fail in the case examined in this article [24]. The comparison is performed by numerical tests executed in the aforementioned operating conditions. The method proposed in [15] is based on Artificial Neural Network (ANN) for the pattern recognition of a set of features extracted from the modulated signal.

The features used by the method proposed in [15] are:

- the maximum of the squared Fourier transform of the normalised signal amplitude;

- the index of the spectrum symmetry around the carrier frequency; and

- the standard deviation of the instantaneous phase.

The first disadvantage of the classifier proposed in [15] with respect to the proposed AMC-EM is that it requires knowledge of the carrier frequency, and this information must be included in the training and in the test phases. This a priori requirement requires that the numerical tests are executed by considering a fixed value of the carrier frequency in order to compare the two methods. In the following section, a carrier frequency of 5.25 $\mathrm{kHz}$ is selected.

The same number of samples and sampling frequency are used to test both the classifiers. After the training of the network, 100 signals for each modulation scheme are classified by the two methods: the proposed one (i.e. the quadratic SVM with features

\begin{tabular}{|c|c|c|c|c|}
\hline \multirow{2}{*}{ True Modulation } & \multicolumn{4}{|c|}{ Predicted Modulation by SVM linear } \\
\hline & AM & PM & FM & SSB \\
\hline $\mathrm{AM}$ & 200 & & & \\
\hline PM & & 200 & & \\
\hline FM & & & 200 & \\
\hline SSB & 1 & & & 199 \\
\hline
\end{tabular}

\begin{tabular}{|c|r|r|r|r|}
\hline \multirow{2}{*}{ True Modulation } & \multicolumn{4}{|c|}{ Predicted Modulation by SVM quadratic } \\
\cline { 2 - 5 } & AM & PM & FM & \multicolumn{1}{c|}{ SSB } \\
\hline AM & 200 & & & \\
\hline PM & & 200 & & \\
\hline FM & & & 200 & \\
\hline SSB & & & & 200 \\
\hline
\end{tabular}

\begin{tabular}{|c|r|r|r|r|}
\hline \multirow{2}{*}{ True Modulation } & \multicolumn{4}{|c|}{ Predicted Modulation by SVM cubic } \\
\cline { 2 - 5 } & \multicolumn{1}{|c|}{ AM } & PM & \multicolumn{1}{c|}{ FM } & \multicolumn{1}{c|}{ SSB } \\
\hline AM & 200 & & & \\
\hline PM & & 200 & & \\
\hline FM & & & 200 & \\
\hline SSB & & & 1 & 199 \\
\hline
\end{tabular}

\begin{tabular}{|c|r|r|r|r|}
\hline \multirow{2}{*}{ True Modulation } & \multicolumn{5}{|c|}{ Predicted Modulation by kNN } \\
\cline { 2 - 5 } & \multicolumn{1}{|c|}{ AM } & PM & \multicolumn{1}{c|}{ FM } & \multicolumn{1}{c|}{ SSB } \\
\hline AM & 200 & & & \\
\hline PM & & 200 & & \\
\hline FM & & 3 & 197 & \\
\hline SSB & 1 & & & 199 \\
\hline
\end{tabular}

Figure 9. Confusion matrix of the classification results with signals not included in the training set.

extracted from the EMD) and the ANN proposed in [15]. Figure 10 shows the overall accuracy of the two methods versus the SNR. If the SNR of the signal is higher than $5 \mathrm{~dB}$, both methods obtain an accuracy of $100 \%$.

For an SNR lower or equal to $5 \mathrm{~dB}$, the $\mathrm{ANN}$ decreases its accuracy with respect to the proposed method.

\subsection{Experimental tests}

In order to evaluate the performance of the proposed AMCEM experimentally, a specific test bed is designed (Figure 11),

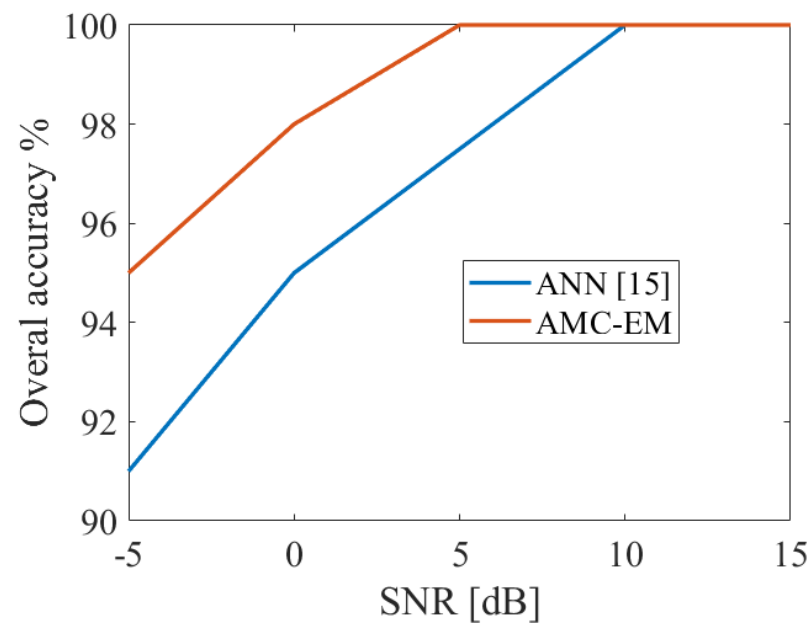

Figure 10. Overall accuracy, in percentage, for the proposed AMC-EM and for the classifier proposed in [15]. 


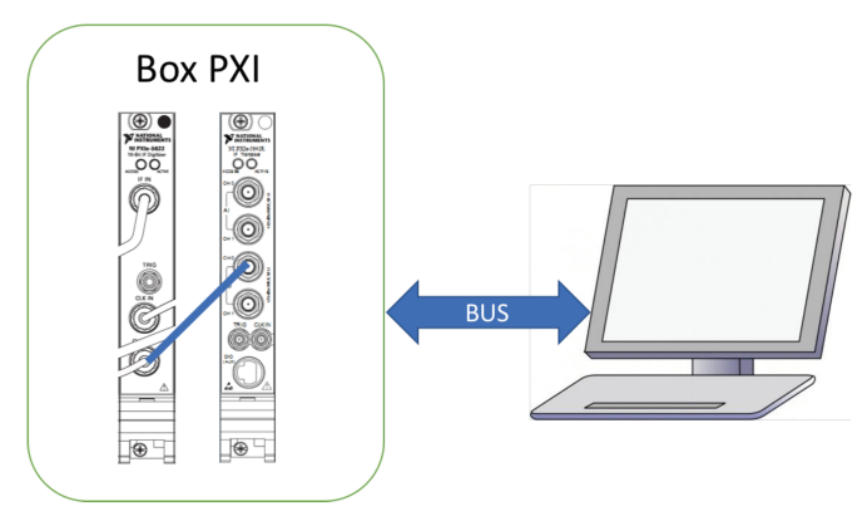

Figure 11. Test bed scheme for the experimental tests.

allowing for the acquisition and analysis of the modulated signals in a wide-band spectrum [31].

The test bed is formed of:

- a PC for the management of the instrumentation and the analysis of the acquired signals;

- a PXI chassis, which includes:

$\circ$ an NI 5622 IF digitiser from National Instruments for the acquisition of the signals; and

- a PXIe-5641R transceiver for signal generation.

Suitable software developed in LabVIEW runs on the PC for the instrumentation setup and the acquisition of the signals. In particular, the LabVIEW software:

- $\quad$ sets up the PXIe-5641R to generate a signal with a carrier frequency of $305.25 \mathrm{kHz}$, a selected modulation, and an established superimposed level of noise;

- $\quad$ triggers the PXIe-5641R to generate the signal; and

- triggers the PXI 5622 to acquire the modulated signal, which is digitally down-converted to have a carrier frequency of $5.25 \mathrm{kHz}$ and is down-sampled to $80 \mathrm{kHz}$.

After the digital down conversion, the LabVIEW software executes the MATLAB code for the classification.

In the tests, 100 signals are used for each modulation scheme. Figure 12 shows the results of these experimental tests by varying the SNR. In particular, 0,5 , and $10 \mathrm{~dB}$ of SNR have been considered. As shown in Figure 12, the experimental results agree with the numerical ones. In particular, the robustness of the AMC-EM is also experimentally assessed for a low value of SNR: at $0 \mathrm{~dB}$, there is a misevaluation of the FM signals of only $2 \%$.

\section{CONCLUSIONS}

In this article, a new automatic analog modulation classifier, the AMC-EM, was presented. The AMC-EM works without the a priori information of the input signals. It analyses the modulated signal by means of the EMD to extract the features that are used by a suitable ML technique for the classification. Selection of the specific ML technique is performed by comparing the performance of the most commonly used ones in the field of signal classification and the quadratic SVM has showed the best performance.

The AMC-EM has been characterised in simulation by considering signals corrupted by additive white Gaussian noise, and it also shows very interesting results for low SNR values. The usefulness of the proposed classifier with respect to the existing ones was assessed by comparing the AMC-EM with another classifier, available in the literature, based on ANN. With respect to other classifiers presented in the literature, the ANN can be

\begin{tabular}{|c|c|c|c|c|c|}
\hline & $\mathbf{A M}$ & PM & FM & SSB \\
\hline \multirow{3}{*}{ 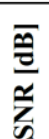 } & 0 & 100 & 100 & 98 & 100 \\
\hline & 5 & 100 & 100 & 100 & 100 \\
\hline & 10 & 100 & 100 & 100 & 100 \\
\hline
\end{tabular}

Figure 12. The number of signals correctly classified by AMC-EM in the experimental test with a SNR equal to $0,5,10 \mathrm{~dB}$.

used with different modulations, such as the AMC-EM. Conversely, the other ones are suitable only for specific modulations. Differently to AMC-EM, the ANN classifier requires the a priori knowledge of the carrier frequency, while the AMC-EM operates in completely blind conditions. Moreover, by testing the two classifiers in the same operating conditions, it is highlighted that the AMC-EM is more robust concerning the influence of noise.

Experimental tests have been carried out to evaluate the AMC-EM with real signals. The experimental results agree with the simulated ones, making AMC-EM a promising method for the automatic classification of the analog signal in typically noisy environments.

\section{REFERENCES}

[1] A. Sengur, Multiclass least-squares support vector machines for analog modulation classification, Expert Syst. Appl. 36(3, 2) (2009), pp. 6681-6685.

[2] J. J. Popoola, R. van Olst, Development and comparative study of effects of training algorithms on performance of artificial neural network based analog and digital automatic modulation recognition, J. Eng. Sci. Technol. Rev. 8(4) (2015), pp. 135-144.

[3] D. L. Carnì, L. De Vito, S. Rapuano, A portable instrument for automatic detection and classification of telecommunication signals, Meas. J. Int. Meas. Confed. 45(2) (2012), pp. 182-189.

[4] L. De Vito, D. D. Napolitano, S. Rapuano, M. Villanacci, D. L. Carnì, A portable automatic digital modulation scanner', Proc. of the 17th Symposium IMEKO TC4 - Measurement of Electrical Quantities, 15th International Workshop on ADC Modelling and Testing, and 3rd Symposium IMEKO TC19 Environmental Measurements, 2010, pp. 445-450.

[5] B. I. Dahap, H. Liao, Advanced algorithm for automatic modulation recognition for analogue \& digital signals, Proc. of the 2015 International Conference on Computing, Control, Networking, Electronics and Embedded Systems Engineering, ICCNEEE 2015, 2016.

[6] X. F. Gao, Y.L. An, Y. Liu, Recognition of the analog modulation modes, Applied Mechanics and Materials, vol. 340. 2013, pp. 773-777.

[7] N. Kim, N. Kehtarnavaz, S. Brown, T. McKinney, Hierarchical classification of modulation signals, Proc. of Robotics, Automation, Control and Manufacturing: Trends, Principles and Applications - The 5th Biannual World Automation Congress, WAC 2002, ISORA 2002, ISIAC 2002 and ISOMA 2002, 2002, vol. 14.

[8] D. A. Amoedo, W. S. Da Silva Jr., E. B. De Lima Filho, Parameter selection for SVM in automatic modulation classification of analog and digital signals, Proc. of the 2014 International Telecommunications Symposium, ITS 2014.

[9] J. J. Popoola, R. Van Olst, Automatic classification of combined analog and digital modulation schemes using feedforward neural network, in Proc. of the IEEE AFRICON Conference, 2011.

[10] E. Balestrieri, P. Daponte, L. De Vito, S. Rapuano, Jitter and its relatives: A critical overview, Proc. of the IEEE Instrumentation and Measurement Technology Conference, 2013, pp. 1141-1146.

[11] M. Petrova, P. Mähönen, A. Osuna, Multi-class classification of analog and digital signals in cognitive radios using support vector 
machines, Proc. of the 7th International Symposium on Wireless Communication Systems, ISWCS'10, 2010.

[12] E. Avci, D. Avci, Using combination of support vector machines for automatic analog modulation recognition, Expert Syst. Appl. 36(2, 2) (2009), pp. 3956-3964.

[13] E. Balestrieri, M. Catelani, L. L. Ciani, S. Rapuano, A. Zanobini, Uncertainty evaluation of DAC time response parameters, Proc. of the IMEKO TC4 International Workshop on ADC Modelling, Testing and Data Converter Analysis and Design 2011, IWADC 2011 and IEEE 2011 ADC Forum, 2011, pp. 278-281.

[14] C.-S. Park, W. Jang, S.-P. Nah, D. Y. Kim, Automatic modulation recognition using support vector machine in software radio applications, Proc. of the International Conference on Advanced Communication Technology, ICACT, 2007, vol. 1.

[15] H. Guldemir, A. Sengur, Online modulation recognition of analog communication signals using neural network, Expert Syst. Appl. 33(1) (2007) pp. 206-214.

[16] M. Richterová, D. Juráěek, A. Mazálek, Modulation classifier of analogue modulated signals based on method of artificial neural networks, Proc. of the International Conference on Applied Electronics 2006, AE, 2006.

[17] A. Baccigalupi, A. Liccardo, The Huang Hilbert Transform for evaluating the instantaneous frequency evolution of transient signals in non-linear systems, Measurement, J. Int. Meas. Confed. 86 (2016), pp. 1-13.

[18] H. Chaudhari, S. L. Nalbalwar, R. Sheth, A review on intrinsic mode function of EMD, Proc. of the International Conference on Electrical, Electronics, and Optimization Techniques, ICEEOT 2016, 2016.

[19] H. Liu, J. Zhang, Y. Cheng, C. Lu, Fault diagnosis of gearbox using empirical mode decomposition and multi-fractal detrended cross-correlation analysis, J. Sound Vib. 385 (2016), pp. 350-371.

[20] Z. Liu, Q. Ying, Z. Luo, Y. Fan, Analysis and research on EEG signals based on HHT algorithm, Proc. of the 6th International Conference on Instrumentation and Measurement, Computer, Communication and Control, IMCCC 2016, 2016.

[21] Y. Lv, R. Yuan, G. Song, Multivariate empirical mode decomposition and its application to fault diagnosis of rolling bearing, Mech. Syst. Signal Process. 81 (2016), pp. 219-234.
[22] P. Nguyen, J.-M. Kim, Adaptive ECG denoising using genetic algorithm-based thresholding and ensemble empirical mode decomposition, Inf. Sci. (Ny). 373 (2016), pp. 499-511.

[23] G. Siracusano F. Lamonaca, R. Tomasello, F. Garescì, A. La Corte, D. L. Carnì, M. Carpentieri, D. Grimaldi, G. Finocchio, A framework for the damage evaluation of acoustic emission signals through Hilbert-Huang transform, Mech. Syst. Signal Process., Vol.75, 2016, pp. 109-122.

[24] Y. H. Tanc, A. Akan, Modulation identification of digital M-ary QAM signals by Hilbert-Huang Transform, Proc. of the IEEE International Conference on Electronics, Circuits, and Systems, 2013.

[25] R. Kannan, S. Ravi, Second-order statistical approach for digital modulation scheme classification in cognitive radio using support vector machine and K-Nearest neighbor classifier, J. Comput. Sci. 9(2) (2013), pp. 235-243.

[26] R. Wang, J.Zhou, J. Chen, Y. Wang, Fast empirical mode decomposition based on Gaussian noises, Proc. of the 3rd International Conference on Mathematics and Computers in Sciences and in Industry, MCSI 2016, 2017.

[27] B. Schölkopf, A. J. Smola, A short introduction to learning with kernels, vol. 2600, Springer, Berlin, Heidelberg, 2003, ISBN 978 3-540-00529-2.

[28] B. Schölkopf, An Introduction to Support Vector Machines, Cambridge University Press, New York, 2003, ISBN 9780521780193.

[29] H. O. Alanazi, A. H. Abdullah, K. N. Qureshi, A critical review for developing accurate and dynamic predictive models using machine learning methods in medicine and health care, J. Med. Syst. 41(4) (2017), pp. 2-10.

[30] R. E. Schapire, Explaining adaboost, in: Empirical inference. Festschrift in honor of Vladimir N. Vapnik, Springer, Berlin, Heidelberg, 2013, ISBN 978-3-642-41135-9, pp. 37-52.

[31] L. Angrisani, F. Bonavolontà, A. Liccardo, R. Schiano Lo Moriello, L. Ferrigno, M. Laracca, G. Miele, Multi-channel simultaneous data acquisition through a compressive samplingbased approach, Meas. J. Int. Meas. Confed. 52(1) (2014) pp. 156172. 